\title{
Acides gras oméga-3 et risque cardiovasculaire
}

\section{Jean DALLONGEVILLE \\ Nadine MARECAUX \\ Philippe AMOUYEL}

Service d'épidémiologie et santé publique, Institut Pasteur de Lille,

Inserm U744,

1 rue du Professeur-Calmette,

59019 Lille cedex,

France

<jean.dallongeville@pasteur-lille.fr>

\begin{abstract}
Linoleic acid (LA) and alpha-linolenic acid (ALA) are precursors of omega-6 and omega-3 fatty acids. Eicosapentaenoic acid (EPA) and docosahexaenoic acid (DHA) are synthesized from ALA. These fatty acids play important role in various biological functions. The effects of omega-3 on cardiovascular risk factors - such as lipoprotein levels, blood pressure and glycemia - are very modest at standard low doses, significant only for large intakes. Randomized trials have shown that oils enriched in EPA and DHA have an undeniable impact on heart rhythm. Consumers of long-chain fatty acid have lower heart rates than controls. Conversely, patients with established arrhythmias do not appear to benefit from treatment with these fatty acids. The observation of food habits in population cohorts helped to defined the effects of rich in ALA, EPA, DHA on the risk of myocardial infarction, coronary heart disease and sudden death. For ALA the results are inconsistent and appropriate randomized clinical trials are still conclude. The effects of EPA and DHA are better established. The meta-analysis of cohort studies shows that consumption of omega-3 long- chain (EPA-DHA) reduces total mortality, death from cardiac causes, sudden death and possibly stroke. The data from prevention trials with omega-3 long-chain showed a decrease in coronary events, including fatal, in secondary prevention. These results are relatively consistent to believe that EPA and DHA intake reduces the fatal complications of myocardial infarction. Clinical trials in primary prevention are still missing. However, the sum of evidence support the recommendation for consumption of fish, EPA and DHA in patients at high risk of coronary event.
\end{abstract}

Key words: $n-3$ fatty acids, fish oil, coronary heart disease, triglycerides, sudden death, arrhythmia

L'ALA est le précurseur de l'acide éicosapentaénoïque (EPA : 20 atomes de carbone - cinq doubles liaisons) et de I'acide docosahexaénoïque (DHA : 22 atomes de carbones - six doubles liaisons). Les données relatives à la conversion de I'ALA en EPA et en DHA chez I'Homme sont controversées. Une partie de I'ALA est métabolisée en EPA, notamment lorsque les apports d'ALA sont importants, mais assez peu en DHA. Par ailleurs, l'EPA et le DHA se trouvent à l'état naturel dans les algues et les lipides des poissons de mer, par où ils entrent dans la chaîne alimentaire de l'Homme.

\section{Acides gras oméga-3 et facteurs de risque cardiovasculaire}

L'infarctus du myocarde est l'aboutissement $d^{\prime}$ un processus complexe initié par des lésions de l'endothélium des artères coronaires et suivi par l'accumulation de lipides dans la paroi vasculaire, la formation d'une plaque d'athérome, l'initiation de réactions inflammatoires favorisant la rupture de la plaque d'athérome et la formation d'un thrombus dans la lumière de l'artère. La formation de la plaque d'athérome et son développement sont facilités par des facteurs de risque métaboliques et hémodyna- miques. Les principaux sont les dyslipidémies (hypercholestérolémie et hypoHDLémie), l'hyperglycémie et l'hypertension artérielle. Les acides gras ont des effets spécifiques sur chacun de ces processus biologiques et facteurs de risque.

Chez l'Homme, l'ingestion de 2 à $3 \mathrm{~g}$ d'EPADHA par jour, généralement sous forme de suppléments, diminue les triglycérides plasmatiques (Harris, 1997) et augmente modérément le HDL et LDL-cholestérol (Mori et Beilin, 2001). Les effets sur les triglycérides plasmatiques sont directement proportionnels à la quantité d'acides gras oméga-3 ingérée. L'augmentation du LDL-cholestérol est faible, en relation directe avec la baisse des triglycérides. Pour des apports plus élevés d'EPA-DHA, le LDL-cholestérol peut diminuer en raison d'une diminution concomitante des apports en acides gras saturés (compensation). De nombreux travaux ont évalué les effets des acides gras oméga- 3 sur la glycémie sans mettre en évidence un effet particulier (favorable ou délétère) sur les indicateurs de l'homéostasie glucidique (Balk et al., 2006a). Enfin, la consommation d'acides gras oméga- 3 est associée à une diminution de la pression artérielle (Geleijnse et al., 2002 ; Dickinson et al., 2006). L'effet des acides gras sur la pression artérielle est modeste mais remarquablement 


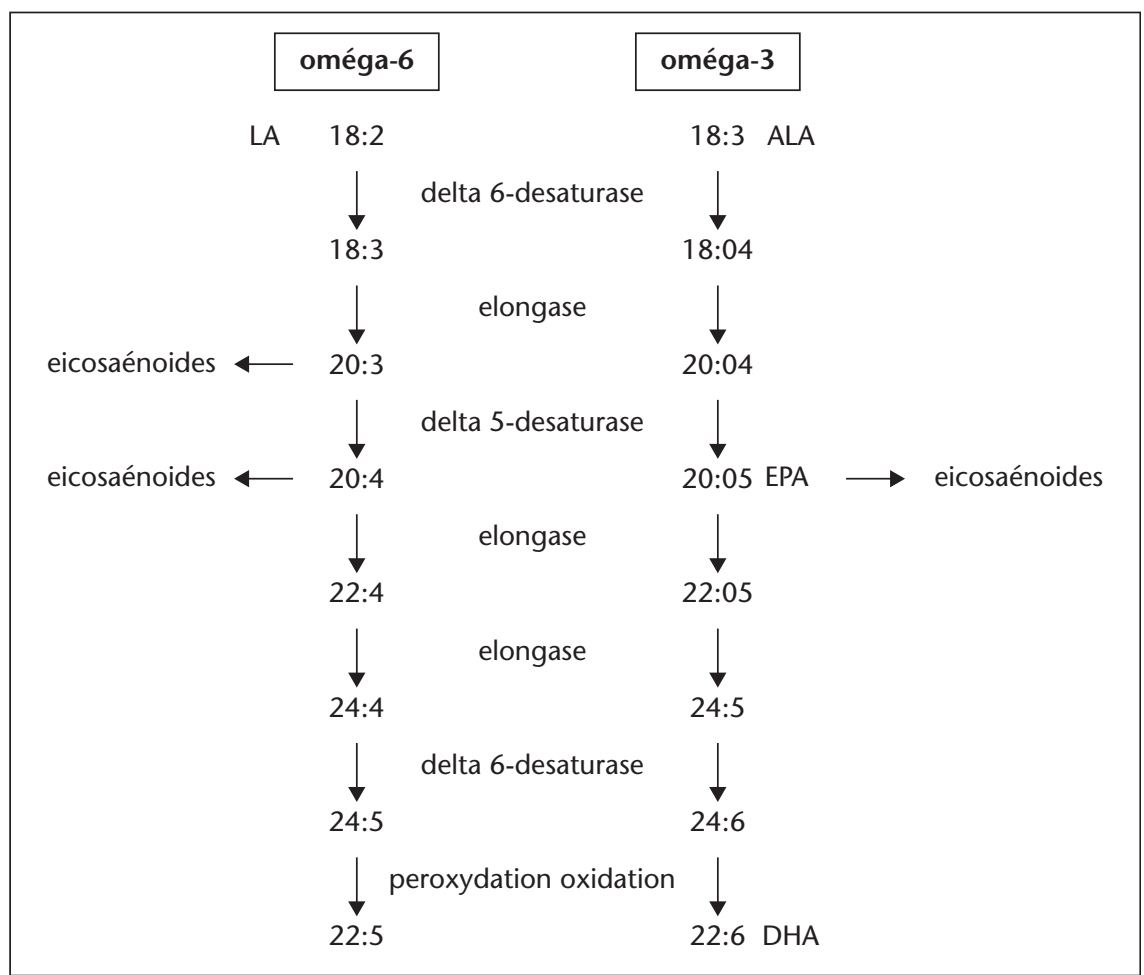

Figure 1. Formation des acides gras dérivés des précurseurs : acide linoléique et alphalinolénique C18:3.

reproductible entre les études. Sur le plan de I'hémostase et de l'aggrégabilité plaquettaire, les résultats sont équivoques. Cependant, des travaux rapportent des résultats concordants pour la diminution des concentrations de fibrine plasmatique. Enfin, des apports importants d'acides gras oméga-3 sont associés à une amélioration des paramètres inflammatoires tels que la CRP et les cytokines pro-inflammatoires. Mais ces effets semblent avoir peu d'impact sur la compliance artérielle ou sur l'athérosclérose carotidienne (Balk et al., 2006b).

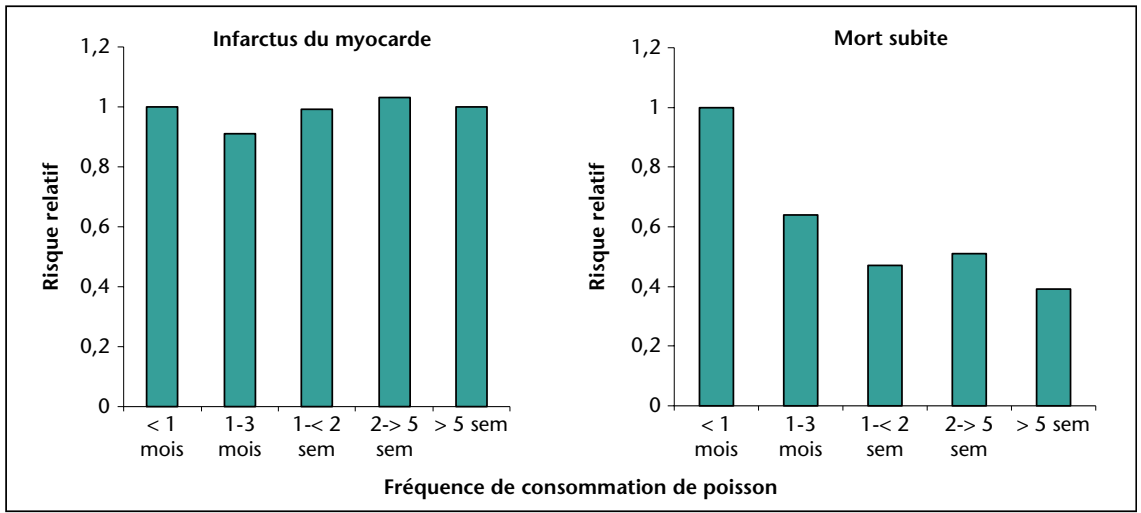

Figure 2. Risque relatif d'infarctus du myocarde et de mort subite (d'après la Physician Health Study. Albert et al. JAMA, 1998). des accidents vasculaires. L'analyse détaillée de ces événements a mis en évidence un bénéfice particulier sur la prévention des accidents fatals et de la mort subite (figure 2).

La méta-analyse des données des cohortes a confirmé ces observations en montrant une relation inverse entre la consommation d'EPA$\mathrm{DHA}$ et la mortalité totale, les décès d'origine cardiaque, les taux de morts subites et possiblement d'accidents vasculaires cérébraux ( $\mathrm{He}$ et al., 2004a, 2004b). Par ailleurs, les enquêtes d'observation ont montré que les bénéfices sur la mortalité étaient observés pour des apports faibles d'acides gras oméga-3 (de l'ordre du gramme par jour), bien inférieurs aux quantités nécessaires pour diminuer les principaux facteurs de risque cardiovasculaire. Ces dernières observations suggèrent que les relations observées sont expliquées par des voies métaboliques différentes des facteurs de risque « classiques ».

\section{Effets des acides oméga-3 sur les événements cardiovasculaires}

Pour conforter les observations épidémiologiques, plusieurs essais thérapeutiques ont évalué, dans des conditions expérimentales contrôlées, les effets de la consommation de poisson ou d'EPA-DHA sur la survenue d'accidents cardiovasculaires (tableau 1).

\section{Relation avec les événements cardiovasculaires ischémiques dans les cohortes}

L'observation des habitudes alimentaires dans des cohortes et leur mise en relation avec la survenue d'événements coronaires a permis d'établir des relations entre la consommation $d^{\prime}$ aliments riches en EPA-DHA et le risque $d^{\prime}$ infarctus. En règle générale, les enquêtes épidémiologiques montrent des relations entre la consommation de poisson et la baisse
Dans l'étude DART (Burr et al., 1989), des patients coronariens bénéficiaient de conseils nutritionnels visant à augmenter leur consommation de poisson (deux fois par semaine) ou recevaient des suppléments d'EPA-DHA. Ces sujets étaient comparés à des témoins qui consommaient peu de poisson. Au terme de l'étude, une diminution des événements cardiovasculaires fatals était observée chez les patients consommateurs de poisson, suggérant un bénéfice après un premier infarctus. Dans DART II, les effets de la consommation de poissons gras (deux fois par semaine) et/ou de suppléments ( $900 \mathrm{mg} / \mathrm{j}$ d'EPA-DHA) sur la survenue d'événements cardiaques étaient évalués chez des patients angineux (Burr et al., 2003). Après trois à six ans de suivi, les taux de décès cardiaque et de mort subite étaient plus élevés dans le groupe «poisson/oméga-3 » que chez les témoins. Les résultats de ce travail doivent cependant être interprétés avec précaution en raison de multiples problèmes méthodologiques qui ont émaillé le déroulement de l'étude.

Dans l'étude GISSI, 11323 patients coronariens étaient randomisés pour recevoir ou non 850 mg d'éthyl ester d'EPA-DHA par jour (Gruppo Italiano per lo Studio della Sopravvivenza nell'Infarto miocardico, 1999). Après 
Tableau 1. Principales caractéristiques des essais de prévention avec des suppléments en EPA-DHA.

\begin{tabular}{|lllll|}
\hline & DART & GISSI & DART II & JELIS \\
\hline Effectif (n) & 2033 & 11300 & 3114 & 18645 \\
\hline Genre & $\mathrm{H} / \mathrm{F}$ & $\mathrm{H} / \mathrm{F}$ & $\mathrm{H}$ & $\mathrm{H} / \mathrm{F}$ \\
\hline Inclusion & Infarctus & Infarctus & Angine & Hypercholestérolémie \\
\hline Âge (ans) & $<70$ & - & $<70$ & $40-75$ \\
\hline Intervention & $\begin{array}{l}200 \text { à } 400 \mathrm{~g} \text { de } \\
\text { poisson/semaine }\end{array}$ & $\begin{array}{l}850 \mathrm{mg} \\
\text { EPA/DHA }\end{array}$ & $\begin{array}{l}\text { 2 parts de poisson/ } \\
\text { semaine ou } \\
\text { suppléments }\end{array}$ & $1,8 \mathrm{~g} / \mathrm{j}$ EPA \\
\hline $\begin{array}{l}\text { Critère de } \\
\text { jugement }\end{array}$ & Décès et cardiopathie & $\begin{array}{l}\text { Décès, } \\
\text { infarctus } \\
\text { et AVC }\end{array}$ & $\begin{array}{l}\text { Décès, infarctus } \\
\text { et AVC }\end{array}$ & $\begin{array}{l}\text { Mort subite, infarctus, } \\
\text { syndrome coronaire } \\
\text { aigu, revascularisation }\end{array}$ \\
\hline Suivi (ans) & 2 & 3,5 & 3,5 & 4,6 \\
\hline
\end{tabular}

Tableau 2. Risque relatif d'événement fatal à non fatal chez les sujets recevant les suppléments d'EPA-DHA dans l'essai de prévention GISSI.

\begin{tabular}{|ll|}
\hline & Risque relatif \\
\hline Décès & $0,80[0,67-0,94]$ \\
\hline Décès cardiovasculaire & $0,65[0,51-0,82]$ \\
\hline Décès coronaire & $0,65[0,51-0,84]$ \\
\hline Mort subite & $0,55[0,55-0,76]$ \\
\hline Évènements non fatals & $0,96[0,76-1,21]$ \\
\hline Autres causes de décès & $0,99[0,75-1,30]$ \\
\hline
\end{tabular}

3,5 années de suivi, les taux de mortalité totale et cardiovasculaire étaient plus bas dans le groupe EPA-DHA que dans le groupe témoins (tableau 2). Cet effet était significatif dès la première année de traitement, notamment pour les événements coronaires fatals (Marchioli et al., 2002).

Enfin, JELIS est un essai de prévention conduit au Japon chez 18645 patients hypercholestérolémiques bénéficiant d'un traitement par une statine avec ou sans EPA $(1,8 \mathrm{~g} / \mathrm{j})$. Dans ce travail, une baisse de $19 \%$ des événements coronaires majeurs (critère combiné de mort subite, infarctus fatal ou non, angor instable, angioplastie et pontage coronaire) était observée chez les patients recevant I'EPA (Yokoyama et al., 2007). La diminution des événements était principalement due à la diminution de l'incidence de l'angor instable et des événements non fatals. II est important de noter que les taux d'événement étaient particulièrement bas dans cette étude conduite au Japon, dans une cohorte majoritairement composée de sujets en prévention primaire. Cette caractéristique explique possiblement la particularité des résultats.

\section{Effets sur le rythme cardiaque et les arythmies}

Depuis le milieu des années 1990, des études expérimentales chez l'animal de laboratoire ont montré que les acides gras oméga- 3 ont des effets particuliers sur la régulation du rythme cardiaque (Kang et Leaf, 1996; London et al., 2007). Ces observations ont stimulé des recherches chez l'Homme, notamment épidémiologiques, et la réalisation d'essais cliniques pour préciser le potentiel antiarythmique des acides gras oméga-3.

Chez le chien, les expériences de fibrillations auriculaires (FA) induites par stimulation vagale ont montré une diminution importante du déclenchement des FA chez les animaux bénéficiant d'un traitement par les acides gras oméga-3 à longues chaînes (EPA et DHA), suggérant un rôle possible dans la prévention de la FA (Sarrazin et al., 2007). Chez l'Homme, les résultats des études épidémiologiques d'observation sur la FA sont équivoques. Certaines ont montré, chez des sujets âgés, que la consommation de poissons grillés ou ébouillantés (par opposition à frits) était associée à une diminution de la survenue des FA (Mozaffarian et al., 2004 ; Frost et Vestergaard, 2005). D'autres enquêtes n'ont pas mis en évidence de diminution des FA chez les consommateurs de poisson ou d'EPA-DHA ne permettant pas de conclure définitivement pour la prévention primaire (Frost et Vestergaard, 2005 ; Brouwer et al., 2006). En revanche, dans un essai randomisé en ouvert, l'administration de $2 \mathrm{~g}$ d'huile de poisson par jour, au décours d'une intervention chirurgicale cardiaque, s'est accompagnée d'une diminution des taux de survenue des FA pendant les suites opératoires (Calo et al., 2005). La fréquence cardiaque est un marqueur du risque de mort subite. Dans les essais randomisés en double insu, la consommation d'huile de poisson s'accompagne d'une diminution de la fréquence cardiaque chez l'Homme (Mozaffarian et al., 2005). Cet effet est particulièrement marqué chez les sujets qui présentent le rythme cardiaque le plus élevé. Ces résultats démontrent un effet propre des acides gras sur le contrôle du rythme et pourraient expliquer une partie des bénéfices observés dans la prévention des morts subites.

La variabilité du rythme cardiaque est un indicateur de l'adaptabilité du système cardiovasculaire aux contraintes physiologiques. Les effets de la consommation d'huile de poisson ou d'EPA-DHA sur ce paramètre ont été évalués dans des essais randomisés en double insu. Les résultats de ces études sont équivoques, ne permettant pas de conclure formellement (Geelen et al., 2004 ; O'Keefe et al., 2006). Cette incertitude reflète en partie I'hétérogénéité des caractéristiques des patients inclus dans les essais de prévention et les difficultés méthodologiques liées au recueil des indicateurs électrocardiographiques de la variabilité du rythme cardiaque.

Chez l'animal de laboratoire, l'ingestion d'huile de poisson ou de suppléments d'EPA-DHA réduit la survenue des tachycardies et des fibrillations ventriculaires induites par ischémie cardiaque (Matthan et al., 2005). Cette observation chez l'animal offre une explication possible aux bénéfices du traitement par les acides gras oméga-3 en prévention secondaire, chez des patients coronariens. Parallèlement à ces observations, plusieurs essais thérapeutiques ont évalué spécifiquement les effets de la consommation d'acides gras oméga-3 sur la survenue de troubles du rythme. Les essais randomisés de prévention des extrasystoles ventriculaires $n^{\prime}$ ont pas mis en évidence $d^{\prime}$ effet favorable ou délétère de l'huile de poisson ou des suppléments d'EPA-DHA (Geelen et al., 2004, 2005). Trois essais randomisés contrôlés (Brouwer et al., 2006; Raitt et al., 2005 ; Jenkins et al., 2008) ont évalué l'effet des suppléments d'EPA-DHA sur la survenue de fibrillations et tachycardies ventriculaires chez des patients bénéficiant d'un stimulateur cardiaque (Leaf et al., 2005). Ces études tirent profit de l'enregistrement des épisodes d'arythmie chez des patients porteurs d'appareillage d'électrostimulation. Les résultats de ces études ne permettent pas de conclure clairement sur le rôle des acides gras oméga-3 dans la prévention des fibrillations et tachycardies ventriculaires (Brouwer et al., 2006). Cependant, compte tenu du petit nombre d'études, des différences méthodologiques, de la diversité des caractéristiques des patients en termes d'antécédent coronaire ou de traitement antiarythmique, il est nécessaire de poursuivre les essais cliniques pour mieux préciser les propriétés antiarythmiques des acides gras oméga-3. 
En conclusion, les essais randomisés ont clairement montré que I'huile de poisson et les acides gras oméga-3 à longues chaînes (EPA et $\mathrm{DHA}$ ) ont un effet indiscutable sur la régulation du rythme cardiaque. En revanche, les patients présentant des troubles du rythme constitués, notamment sur des cœurs non ischémiques, ne semblent pas tirer profit du traitement par ces acides gras pour prévenir les troubles du rythme.

\section{Conclusion}

La relation entre la consommation d'aliments riches en acides gras oméga- 3 ou de suppléments d'EPA-DHA et la survenue d'accidents coronaires fatals est maintenant bien établie. Ces acides gras pourraient agir en diminuant la survenue des troubles du rythme liés à l'ischémie myocardique. En revanche, les connaissances sur les effets des acides gras oméga- 3 chez des patients présentant des troubles du rythme constitués sont encore insuffisantes. Ainsi, la consommation de poisson, d'EPA-DHA est recommandée chez les sujets à risque élevé $\mathrm{d}^{\prime}$ accident coronaire ou de récidive vasculaire. Les apports nécessaires pour prévenir les accidents fatals sont de l'ordre de $750 \mathrm{mg} / \mathrm{j}$.

\section{RÉFÉRENCES}

Balk EM, Lichtenstein AH, Chung M, et al. Effects of omega-3 fatty acids on serum markers of cardiovascular disease risk: a systematic review. Atherosclerosis 2006a ; 189 : 19-30.

Balk EM, Lichtenstein AH, Chung $M$, et al. Effects of omega-3 fatty acids on coronary restenosis, intimamedia thickness, and exercise tolerance: a systematic review. Atherosclerosis 2006b ; 184 : 237-46.

Brouwer IA, Zock PL, Camm AJ, et al. Effect of fish oil on ventricular tachyarrhythmia and death in patients with implantable cardioverter defibrillators: the Study on Omega-3 Fatty Acids and Ventricular Arrhythmia (SOFA) randomized trial. JAMA 2006; 295 : 2613-9.

Burr ML, Fehily AM, Gilbert JF, et al. Effects of changes in fat, fish, and fibre intakes on death and myocardial reinfarction: diet and reinfarction trial (DART). Lancet $1989 ; 2$ : 757-61.
Burr ML, Ashfield-Watt PA, Dunstan FD, et al. Lack of benefit of dietary advice to men with angina: results of a controlled trial. Eur / Clin Nutr 2003; 57 : 193-200.

Calo L, Bianconi L, Colivicchi F, et al. n-3 fatty acids for the prevention of atrial fibrillation after coronary artery bypass surgery: a randomized, controlled trial. J Am Coll Cardiol 2005 ; 45 : 1723-8.

Dickinson HO, Mason JM, Nicolson DJ, et al. Lifestyle interventions to reduce raised blood pressure: a systematic review of randomized controlled trials. J Hypertens $2006 ; 24: 215-33$.

Frost L, Vestergaard P. n-3 fatty acids consumed from fish and risk of atrial fibrillation or flutter: the Danish Diet, Cancer, and Health Study. Am / Clin Nutr 2005 ; $81: 50-4$.

Geelen A, Brouwer IA, Zock PL, Katan MB. Antiarrhythmic effects of $n-3$ fatty acids: evidence from human studies. Curr Opin Lipidol 2004 ; 15 : 25-30.

Geelen A, Brouwer IA, Schouten EG, et al. Effects of $\mathrm{n}-3$ fatty acids from fish on premature ventricular complexes and heart rate in humans. Am / Clin Nutr $2005 ; 81: 416-20$.

Geleijnse JM, Giltay EJ, Grobbee DE, Donders AR, Kok FJ. Blood pressure response to fish oil supplementation: metaregression analysis of randomized trials. J Hypertens $2002 ; 20$ : 1493-9.

Gruppo italiano per lo studio della sopravvivenza nell'infarto miocardico. Dietary supplementation with $\mathrm{n}-3$ polyunsaturated fatty acids and vitamin $\mathrm{E}$ after myocardial infarction: results of the GISSIPrevenzione trial. Lancet 1999 ; 354 : 447-55.

Harris WS. n-3 fatty acids and serum lipoproteins: human studies. Am / Clin Nutr 1997 ; 65 : 1645S1654 S.

He K, Song Y, Daviglus ML, et al. Accumulated evidence on fish consumption and coronary heart disease mortality: a meta-analysis of cohort studies. Circulation 2004a ; 109 : 2705-11.

He K, Song Y, Daviglus ML, et al. Fish consumption and incidence of stroke: a meta-analysis of cohort studies. Stroke 2004b ; 35 : 1538-42.

Jenkins DJ, Josse AR, Beyene J, et al. Fish-oil supplementation in patients with implantable cardioverter defibrillators: a meta-analysis. CMAJ 2008; 178 : 157-64.

Kang JX, Leaf A. Antiarrhythmic effects of polyunsaturated fatty acids. Recent studies. Circulation 1996 ; $94: 1774-80$.
Leaf A, Albert CM, Josephson M, et al. Prevention of fatal arrhythmias in high-risk subjects by fish oil n-3 fatty acid intake. Circulation $2005 ; 112$ : 2762-8.

London B, Albert C, Anderson ME, et al. Omega-3 fatty acids and cardiac arrhythmias: prior studies and recommendations for future research: a report from the National Heart, Lung, and Blood Institute and Office of Dietary Supplements Omega-3 Fatty Acids and their Role in Cardiac Arrhythmogenesis Workshop. Circulation 2007 ; 116 : e320-35.

Marchioli R, Barzi F, Bomba E, et al. Early protection against sudden death by $n-3$ polyunsaturated fatty acids after myocardial infarction: time-course analysis of the results of the Gruppo Italiano per lo Studio della Sopravvivenza nell'Infarto Miocardico (GISSI)Prevenzione. Circulation 2002 ; 105 : 1897-903.

Matthan NR, Jordan H, Chung M, et al. A systematic review and meta-analysis of the impact of omega-3 fatty acids on selected arrhythmia outcomes in animal models. Metabolism 2005 ; 54 : 1557-65.

Mori TA, Beilin LJ. Long-chain omega-3 fatty acids, blood lipids and cardiovascular risk reduction. Curr Opin Lipidol $2001 ; 12$ : 11-7.

Mozaffarian D, Psaty BM, Rimm EB, et al. Fish intake and risk of incident a trial fibrillation. Circulation $2004 ; 110$ : 368-73.

Mozaffarian D, Geelen A, Brouwer IA, et al. Effect of fish oil on heart rate in humans: a meta-analysis of randomized controlled trials. Circulation $2005 ; 112$ : 1945-52.

O'Keefe Jr JH, Abuissa H, Sastre A, Steinhaus DM, Harris WS. Effects of omega- 3 fatty acids on resting heart rate, heart rate recovery after exercise, and heart rate variability in men with healed myocardial infarctions and depressed ejection fractions. Am / Cardiol 2006 ; 97 : 1127-30.

Raitt MH, Connor WE, Morris C, et al. Fish oil supplementation and risk of ventricular tachycardia and ventricular fibrillation in patients with implantable defibrillators: a randomized controlled trial. JAMA 2005 ; 293 : 2884-91.

Sarrazin JF, Comeau G, Daleau P, et al. Reduced incidence of vagally induced atrial fibrillation and expression levels of connexins by $n-3$ polyunsaturated fatty acids in dogs. I Am Coll Cardiol 2007 ; 50 : 1505-12.

Yokoyama M, Origasa H, Matsuzaki M, et al. Effects of eicosapentaenoic acid on major coronary events in hypercholesterolaemic patients (JELIS): a randomised open-label, blinded endpoint analysis. Lancet 2007 ; 369 : 1090-8. 